\title{
Patients with Borderline Personality Disorder and \\ Lifespan Bipolar Disorder (Borderpolar): A Descriptive and Comparative Study
}

\section{Introduction}

\section{Mark Zimmerman, Caroline Balling, Iwona Chelminski, and Kristy Dalrymple \\ Department of Psychiatry and Human Behavior, Brown Medical School, \\ Rhode Island Hospital, Providence, RI, United States}

Bipolar disorder and borderline personality disorder (BPD) are each significant public health problems. Reviews have focused attention on differential diagnosis. While there is a burgeoning literature comparing patients with BPD and bipolar disorder, much less research has characterized patients with both disorders.

The goal of the present report from the Rhode Island Methods to Improve Diagnostic Assessment and Services (MIDAS) project was to compare outpatients with both BPD and bipolar disorder to patients with BPD without bipolar disorder and patients with bipolar disorder without BPD.

\section{Methods}

Outpatients presenting for treatment were evaluated with semi-structured interviews. The focus of the present study is the 517 patients with both bipolar disorder and BPD ( $n=59)$, BPD without bipolar disorder $(n=330)$, and bipolar disorder without BPD $(n=128)$.

\section{Results}

Compared to patients with bipolar disorder, patients with bipolar disorder and BPD had more comorbid disorders, psychopathology in their family, childhood trauma, suicidality, hospitalizations, time unemployed, and likelihood of receiving disability payments. The added presence of bipolar disorder in patients with BPD was associated with more posttraumatic stress disorder in the patients as well as their family, more bipolar disorder and substance use disorders in their first-degree relatives, more childhood trauma, more unemployment and disability, more suicide attempts, and more hospitalizations.

\section{Discussion}

Patients with both bipolar disorder and BPD represent a group with severe psychosocial morbidity who are often unemployed, suicidal, and utilize more costly forms of health care services. Efforts to identify effective approaches towards treating these patients have been minimal and are needed.

Table 1 Demographic Characteristics

\begin{tabular}{|c|c|c|c|c|c|c|c|c|c|c|}
\hline & \multicolumn{2}{|c|}{$\begin{array}{l}\text { Bipolar } \\
(\mathrm{n}=128)\end{array}$} & \multicolumn{2}{|c|}{$\begin{array}{c}\text { Bipolar/BPD } \\
(n=59)\end{array}$} & \multicolumn{2}{|c|}{$\begin{array}{c}\mathrm{BPD} \\
(\mathrm{n}=330)\end{array}$} & \multicolumn{2}{|c|}{$\begin{array}{c}\text { Bipolar/BPD } \\
\text { vs Bipolar }\end{array}$} & \multicolumn{2}{|c|}{$\begin{array}{c}\text { Bipolar/BPD vs } \\
\text { BPD }\end{array}$} \\
\hline & $\mathrm{N}$ & $\%$ & $\mathrm{~N}$ & $\%$ & $\mathrm{~N}$ & $\%$ & $\chi^{2}$ & $p$ & $\chi^{2}$ & $p$ \\
\hline Gender & & & & & & & 2.33 & n.s. & 0.17 & n.s. \\
\hline Male & 54 & 42.2 & 18 & 30.5 & 92 & 27.9 & & & & \\
\hline Female & 74 & 57.8 & 41 & 69.5 & 238 & 72.1 & & & & \\
\hline Education & & & & & & & 4.12 & n.s. & 0.18 & n.s. \\
\hline Less than high school & 8 & 6.3 & 5 & 8.5 & 33 & 10.0 & & & & \\
\hline Graduated high school & 83 & 64.8 & 45 & 76.3 & 244 & 73.9 & & & & \\
\hline Graduated college & 37 & 28.9 & 9 & 15.3 & 53 & 16.1 & & & & \\
\hline Marital status & & & & & & & 9.21 & n.s. & 7.56 & n.s. \\
\hline Married & 52 & 40.6 & 12 & 20.3 & 74 & 22.4 & & & & \\
\hline Living with someone & 5 & 3.9 & 3 & 5.1 & 38 & 11.5 & & & & \\
\hline Widowed & 0 & 0 & 1 & 1.7 & 2 & 0.6 & & & & \\
\hline Separated & 9 & 7.0 & 6 & 10.2 & 15 & 4.5 & & & & \\
\hline Divorced & 20 & 15.6 & 12 & 20.3 & 45 & 13.6 & & & & \\
\hline Never married & 42 & 32.8 & 25 & 42.4 & 156 & 47.3 & & & & \\
\hline & Mean & SD & Mean & SD & Mean & SD & $t$ & & & $\mathrm{p}$ \\
\hline Age & 37.5 & 12.6 & 33.8 & 9.1 & 32.4 & 10.6 & 1.98 & .05 & 1.01 & n.s. \\
\hline
\end{tabular}

Table 2 Frequency of Current DSM-IV Axis I Disorders

\begin{tabular}{|c|c|c|c|c|c|c|c|c|c|c|}
\hline \multirow[b]{2}{*}{ Disorder } & \multicolumn{2}{|c|}{$\begin{array}{l}\text { Bipolar } \\
(n=128)\end{array}$} & \multicolumn{2}{|c|}{$\begin{array}{c}\text { Bipolar/BPD } \\
(n=59)\end{array}$} & \multicolumn{2}{|c|}{$\begin{array}{c}\text { BPD } \\
(n=330)\end{array}$} & \multicolumn{2}{|c|}{$\begin{array}{l}\text { Bipolar/BPD } \\
\text { vs Bipolar }\end{array}$} & \multicolumn{2}{|c|}{$\begin{array}{c}\text { Bipolar/BPD vs } \\
\text { BPD }\end{array}$} \\
\hline & $\mathrm{N}$ & $\%$ & $\mathrm{~N}$ & $\%$ & $\mathrm{~N}$ & $\%$ & $\chi^{2}$ & $\mathrm{p}$ & $\chi^{2}$ & $p$ \\
\hline \multicolumn{11}{|l|}{ Anxiety disorders } \\
\hline Panic w/ or w/out agoraphobia & 28 & 21.9 & 19 & 32.2 & 90 & 27.3 & 2.29 & n.s. & 0.60 & \\
\hline Specific phobia & 18 & 14.1 & 15 & 25.4 & 80 & 24.2 & 3.59 & n.s. & 0.04 & n.s. \\
\hline Social phobia & 49 & 38.3 & 29 & 49.2 & 159 & 48.2 & 1.96 & n.s. & 0.02 & \\
\hline Postraumatic stress disorder & 12 & 9.4 & 29 & 49.2 & 86 & 26.1 & 37.33 & .001 & 12.82 & .001 \\
\hline Obsess & 13 & 10.2 & 17 & 28.8 & 40 & 12.1 & 10.44 & .001 & 11.15 & .001 \\
\hline Generalized anxiety disorde & 43 & 33.6 & 18 & 30.5 & 107 & 32.4 & 0.18 & n.s. & 0.08 & n.s. \\
\hline \multicolumn{11}{|l|}{ Substance use disorders } \\
\hline Alcohol abuse/dependence & 8 & 6.3 & 5 & 8.5 & 62 & 18.8 & 0.31 & & 3.73 & \\
\hline Drug a & 6 & 4.7 & 8 & 13.6 & 43 & 13.0 & 4.59 & .05 & 0.01 & n.s. \\
\hline Any substance use disorder & 14 & 10.9 & 13 & 22.0 & 84 & 25.5 & 4.03 & .05 & 0.31 & n.s. \\
\hline Any eating disorder & 10 & 7.8 & 5 & 8.5 & 28 & 8.5 & 0.02 & n.s. & 0.00 & n.s. \\
\hline & 8 & 6.3 & 10 & 16.9 & 51 & 15.5 & 5.31 & .05 & 0.09 & n.s. \\
\hline \multirow{2}{*}{$\begin{array}{l}\text { Any impulse control disorder } \\
\text { Three or more Axis I disorders }\end{array}$} & 20 & 15.6 & 16 & 27.1 & 73 & 22.1 & 3.42 & n.s. & 0.71 & n.s. \\
\hline & 57 & 44.5 & 42 & 71.2 & 261 & 79.1 & 11.52 & .001 & 1.82 & 1.5 \\
\hline
\end{tabular}

Table 3 DSM-IV Personality Disorder Dimensional Scores

\begin{tabular}{|c|c|c|c|c|c|c|c|c|c|c|}
\hline \multirow[b]{2}{*}{ Personality Disorder } & \multicolumn{2}{|c|}{ Bipolar } & \multicolumn{2}{|c|}{ Bipolar/BPD } & \multicolumn{2}{|c|}{ BPD } & \multicolumn{2}{|c|}{$\begin{array}{l}\text { Bipolar/BPD } \\
\text { vs Bipolar }\end{array}$} & \multicolumn{2}{|c|}{$\begin{array}{c}\text { Bipolar/BPD } \\
\text { vs BPD }\end{array}$} \\
\hline & Mean & SD & Mean & SD & Mean & SD & & $\mathrm{p}$ & $t$ & $\mathrm{p}$ \\
\hline Paranoida & 0.9 & 1.5 & 2.1 & 1.7 & 1.8 & 1.6 & 3.47 & .01 & 0.89 & n.s. \\
\hline Schizoida & 0.5 & 0.8 & 0.7 & 0.9 & 0.6 & 0.9 & 1.22 & n.s. & 0.63 & n.s. \\
\hline Schizotypala & 0.6 & 0.9 & 0.9 & 1.3 & 0.9 & 1.2 & 1.43 & n. & 0.14 & n.s. \\
\hline Antisociall & 0.5 & 1.1 & 1.7 & 1.9 & 1.2 & 1.5 & 4.66 & .001 & 1.73 & n.s. \\
\hline Histrionic ${ }^{a}$ & 1.0 & 1.5 & 1.7 & 1.7 & 1.4 & 1.3 & 2.16 & .05 & 1.07 & n.s. \\
\hline Narcissistica & 1.0 & 1.5 & 1.8 & 1.8 & 1.7 & 1.6 & 2.34 & .05 & 0.47 & n.s. \\
\hline Avoidant ${ }^{\mathrm{a}}$ & 1.4 & 2.1 & 2.4 & 2.2 & 2.1 & 2.2 & 2.20 & .05 & 0.78 & n.s. \\
\hline Dependenta & 0.6 & 1.2 & 1.5 & 1.7 & 1.7 & 1.8 & 3.11 & .01 & 0.60 & n.s. \\
\hline Obsessive-compulsivea & 1.6 & 1.4 & 2.0 & 1.6 & 1.8 & 1.3 & 1.45 & n.s. & 0.92 & n.s. \\
\hline
\end{tabular}

Table 4 Morbid Risks for Psychiatric Disorders in First-Degree Relatives

\begin{tabular}{|c|c|c|c|c|c|c|c|c|c|c|}
\hline & \multicolumn{2}{|c|}{$\begin{array}{l}\text { Bipolar } \\
(n=683)\end{array}$} & \multicolumn{2}{|c|}{$\begin{array}{c}\text { Bipolar/BPD } \\
(n=279)\end{array}$} & \multicolumn{2}{|c|}{$\begin{array}{c}\text { BPD } \\
(n=1506)\end{array}$} & \multicolumn{2}{|c|}{$\begin{array}{c}\text { Bipolar//BPD } \\
\text { vs Bipolar }\end{array}$} & \multicolumn{2}{|c|}{$\begin{array}{c}\text { BipolarlBPD } \\
\text { vs BPD }\end{array}$} \\
\hline & $\begin{array}{l}\text { Relative } \\
\text { eltat }\end{array}$ & Morbid & & Morbid & & Morbia & & & & \\
\hline iety Disorder & & $\frac{k(\%)}{5.5}$ & $\begin{array}{c}\text { at IIsk } \\
196.0\end{array}$ & & & & & & & \\
\hline & & 22.9 & & 2.12 & & 27.3 & & & & \\
\hline & & 6.5 & & 3 & & 3.4 & 6.34 & .01 & 33.50 & - 001 \\
\hline & & 4.5 & 163.5 & 5.5 & 1006.5 & 5.3 & 0.45 & n.s. & 0.03 & n.s. \\
\hline nime & & 1.5 & . & 2.2 & 1010 & 1.1 & 0.52 & n..s. & 2.40 & n..s. \\
\hline & & $\begin{array}{l}1.4 \\
.2\end{array}$ & & 9.0 & & 2.99 & & & 22.92 & .001 \\
\hline & 542 & 0.6 & 207 & 1.9 & 1980.0 & 09 & 400 & 05 & 201 & $\begin{array}{l}n . s . \\
n . s .\end{array}$ \\
\hline & & 17.0 & & 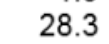 & & 20.5 & 15.71 & 001 & 8.28 & .01 \\
\hline Diso & 490.5 & 8.2 & 186.5 & 20.4 & 1121.5 & 10.6 & 28.66 & 001 & 21.06 & 001 \\
\hline
\end{tabular}

Table 5 Clinical Characteristics

\begin{tabular}{|c|c|c|c|c|c|c|c|c|c|c|}
\hline & \multicolumn{2}{|c|}{$\begin{array}{l}\text { Bipolar } \\
(n=128)\end{array}$} & \multicolumn{2}{|l|}{ Bipolala } & \multicolumn{2}{|c|}{$\begin{array}{l}\text { BPD } \\
(n=330)\end{array}$} & \multicolumn{2}{|c|}{$\begin{array}{l}\text { Bipolar/BPD } \\
\text { vs Bipolar }\end{array}$} & \multicolumn{2}{|c|}{$\begin{array}{l}\text { Bipolar/BPD } \\
\text { vs BPD }\end{array}$} \\
\hline linical Characteristic & Mean & & Mean & & $\begin{array}{l}\text { Mean } \\
110\end{array}$ & 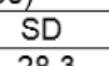 & & $p$ & & \\
\hline & 2 & $\begin{array}{l}1.2 \\
1.5\end{array}$ & $\begin{array}{r}r 9.1 \\
3.0 \\
34\end{array}$ & $\begin{array}{r}46.8 \\
1.2 \\
1.3\end{array}$ & $\begin{array}{l}1,1.9 \\
2.8 \\
3.2\end{array}$ & $\begin{array}{l}8.3 \\
1.0 \\
1.3\end{array}$ & & & & \\
\hline & 1. & $\begin{array}{l}1.5 \\
1.3\end{array}$ & 2.4 & 1.4 & 2.3 & 1.4 & & .001 & & \\
\hline & & 1.5 & 2 & $\begin{array}{l}1.4 \\
1.5\end{array}$ & 2.8 & $\begin{array}{l}1.4 \\
1.5\end{array}$ & & $\begin{array}{l}\text { n.s. } \\
n \text { n.s. }\end{array}$ & & \\
\hline & & & 8 & r. & 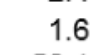 & 1.5 & & 1 & & \\
\hline ore: & $\begin{array}{r}43.6 \\
80\end{array}$ & $\begin{array}{l}18.3 \\
4.9\end{array}$ & $\begin{array}{l}60.6 \\
11.7\end{array}$ & $\begin{array}{l}20.4 \\
6.2\end{array}$ & $\begin{array}{c}50.1 \\
9.3\end{array}$ & $\begin{array}{l}18.7 \\
5.4\end{array}$ & & $\begin{array}{l}.001 \\
.001\end{array}$ & & \\
\hline & & 1.6 & 8.4 & 2.5 & 0.0 & 2.1 & & .01 & & \\
\hline & 12. & & 2.2 & $\begin{array}{l}6.2 \\
4.7\end{array}$ & $\begin{array}{l}10.7 \\
10.2\end{array}$ & 5.7 & & 01 & & \\
\hline & & & & & & & & & & \\
\hline
\end{tabular}

Table 6 Psychosocial Morbidity

\begin{tabular}{|c|c|c|c|c|c|c|c|c|c|c|}
\hline \multirow[b]{2}{*}{ Morbidity Indicator } & \multicolumn{2}{|c|}{$\begin{array}{l}\text { Bipolar } \\
(n=128)\end{array}$} & \multicolumn{2}{|c|}{$\begin{array}{c}\text { Bipolar/BPD } \\
(n=59)\end{array}$} & \multicolumn{2}{|c|}{ 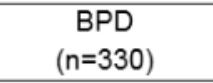 } & \multicolumn{2}{|c|}{$\begin{array}{c}\text { Bipolar/BPD } \\
\text { vs Bipolar }\end{array}$} & \multicolumn{2}{|c|}{$\begin{array}{c}\text { Bipolar/BPD } \\
\text { vs BPD }\end{array}$} \\
\hline & Mean & SD & Mean & SD & Mean & SD & & $p$ & & $\mathrm{p}$ \\
\hline No. current psychia & 2.4 & 1.7 & 4.3 & 2.0 & 5.0 & 2.1 & 6.61 & .001 & 2.30 & .05 \\
\hline Global & 49.0 & 7.6 & 43.3 & 8.7 & 46. & 9 & 4.5 & .00 & 2.68 & .01 \\
\hline Current. & 3.6 & 1.2 & 4.2 & 1.0 & 3.9 & 1.2 & 2.46 & .05 & 1.60 & n.s. \\
\hline \multirow[t]{2}{*}{ Adolescent social functioning } & 2.8 & 1.1 & 3.4 & 1.1 & 3.2 & 1.1 & 3.31 & .01 & 1.65 & n.s. \\
\hline & $\%$ & $\mathrm{n}$ & $\%$ & $n$ & $\%$ & $\mathrm{n}$ & $x^{2}$ & $\mathrm{p}$ & $\chi^{2}$ & \\
\hline hron & 0.7 & 12 & 28.8 & 15 & 9.6 & 28 & 8.49 & .01 & & 1 \\
\hline Persistent unemployment $(n=112,52,291)$ & 22.3 & 25 & 51 & 27 & 24.4 & 71 & 13.84 & .001 & 16.38 & .001 \\
\hline GAF $<$ & 5 & 57 & ( & 3 & 47.4 & 156 & 13.05 & .001 & 12.99 & .001 \\
\hline Temp & 21.4 & 12 & 46.4 & 13 & 11.2 & 17 & 5.58 & .05 & 21.15 & .001 \\
\hline $\mathrm{rm}$ & 8.9 & 5 & 17.9 & 5 & 18.5 & $2 \varepsilon$ & 1.4 & n.s. & 0.01 & n.s. \\
\hline & 33. & 21 & & & & & & & & \\
\hline & 28.1 & 36 & 61. & 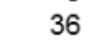 & 47.0 & 155 & 18. & .001 & 5 & 05 \\
\hline & 18.3 & 23 & 32. & 19 & 35.5 & 116 & 4.4 & .05 & 0.24 & \\
\hline ory of psychiatric hospi & 46.1 & 59 & 64.4 & 38 & 42.4 & 140 & 5.43 & .05 & 9.75 & \\
\hline
\end{tabular}

